\title{
Dealiasing Doppler Velocities Measured by a Bistatic Radar Network during a Downburst-Producing Thunderstorm
}

\author{
KATJA Friedrich ANd Olivier CAUMOnT* \\ Institut fuer Physik der Atmosphaere, Deutsches Zentrum fuer Luft-und Raumfahrt (DLR), Oberpfaffenhofen, Wessling, Germany
}

(Manuscript received 25 July 2003, in final form 21 November 2003)

\begin{abstract}
The object of this paper was to develop an automated dealiasing scheme that dealiases Doppler velocities measured by a bistatic Doppler radar network. The particular network consists of the C-band polarimetric diversity Doppler radar, POLDIRAD, and three passive receivers located at remote sites. The wind components, independent but measured simultaneously, are then merged to a horizontal wind vector field. In order to dealias these independent wind components separately, the real-time four-dimensional Doppler dealiasing scheme (4DD) developed by James and Houze was modified. In altering 4DD, the main difficulties arose from dealiasing bistatically measured Doppler velocities, the spatial data inhomogeneity, and to a lesser extent, from the small spatial coverage of bistatic data due to the limited size of the bistatic antenna's aperture. Furthermore, an internal dealiasing algorithm was added to 4DD that uses the full wind vector information to optimize dealising of small isolated cells. Because the determination of microphysical and dynamical parameters requires alternating or fixed polarization bases, respectively, two different scanning strategies are developed to determine these parameters effectively during both slowly and rapidly evolving weather events. An example is presented of dealiasing monostatically and bistatically measured Doppler velocities which were acquired using both scanning modes to observe a downburst-producing thunderstorm.
\end{abstract}

\section{Introduction}

Doppler radar systems sample Doppler velocity and reflectivity over a horizontal range of up to $250 \mathrm{~km}$, with a spatial resolution that is typically several hundred meters by $1^{\circ}$ azimuth and a temporal resolution within minutes. There is a limit to the extent to which velocity can be measured unambiguously by a Doppler radar system. One major problem lies in the so-called aliasing of velocities. The Doppler velocity is normally derived from the difference in phase shift (Doppler frequency) between two successive pulses leading to a time series consisting of a discrete number of samples. Hereby, only the basic fundamental Doppler frequency can be derived unambiguously from this time series but not higherorder harmonic frequencies. More information on dealiasing together with pulse sampling can be found for instance in Doviak and Zrnić (1984) and Keeler and Passarelli (1990).

Monostatic Doppler radar systems measure only the

\footnotetext{
* Current affiliation: Météo-France, CNRM/GMME, Toulouse, France.

Corresponding author address: Katja Friedrich, DLR-Institut fuer Physik der Atmosphaere, Oberpfaffenhofen, 82234 Wessling, Germany.

E-mail: katja.friedrich@dlr.de
}

wind vector component along the transmitting direction. Since the demand for wind vector fields has been increased in the fields of scientific research and operational forecasting, various techniques have been developed to measure or retrieve the full wind vector. The wind vector can be determined from measurements, for instance, if a region is monitored by several monostatic Doppler radars (henceforth monostatic multiple-Doppler radar network). A multiple-Doppler analysis based on a least squares estimation can then be applied (Ray et al. 1978). An alternative to using monostatic multipleDoppler radar networks is to install several passive receivers (henceforth bistatic receivers) at remote sites around one monostatic Doppler radar (henceforth bistatic multiple-Doppler radar network). With such a system not only costs can be significantly reduced but also the interpolation discrepancies of each Doppler velocity measurement in time and space can be made negligible. Nevertheless, irrespective of the Doppler radar system velocities can only be measured unambiguously within the Nyquist velocity interval.

While a number of dealiasing concepts for monostatic Doppler radar data have already been developed since the late 1970s (see, i.e., James and Houze 2001; Tabary et al. 2001, for an overview), no dealiasing scheme has been developed to correct data measured by bistatic receivers. In order to dealias monostatically and bistatically measured Doppler velocities at the same time, we 
modified the real-time four-dimensional Doppler dealiasing scheme (4DD) developed by James and Houze (2001). This algorithm uses the four-dimensionality of the Doppler radar data-that is, the three spatial dimensions along with the time dimension, to constrain the dealiasing. The effectiveness was proven during the Mesoscale Alpine Program (MAP) in 1999 (see Binder et al. 1995; Bougeault 2001) while dealiasing the operational Doppler radar data stream, for instance, from the Meteo Swiss's Monte Lema radar (Joss et al. 1998), which operated at a Nyquist velocity of $8.27 \mathrm{~m} \mathrm{~s}^{-1}$ within complex terrain.

The Deutsches Zentrum für Luft- und Raumfahrt (DLR) in Oberpfaffenhofen (OP) southwest of Munich in southern Germany operates the monostatic polarimetric diversity Doppler radar system, POLDIRAD, additionally equipped with three bistatic receivers. The system is a C-band radar transmitting at a frequency of $5.5 \mathrm{GHz}(\lambda=5.45 \mathrm{~cm})$. The pulse repetition frequency (PRF) is chosen typically to be $1200 \mathrm{~Hz}$, which leads to a Nyquist velocity of $16.35 \mathrm{~m} \mathrm{~s}^{-1}$ and a maximum range of $125 \mathrm{~km}$ (for more details, see section 4).

The objective of this paper is to present the automated dealiasing of bistatically measured Doppler velocities and an optimized scanning strategy. Two scanning modes, which base on varying sampling times for Doppler velocity and reflectivity, were set up in order to observe both microphysical and dynamical parameters simultaneously within rapidly evolving systems and with a dense spatial resolution within slowly evolving systems. Microphysical parameters derived from polarimetric measurements require varying transmitting and receiving polarization bases, while efficient multipleDoppler measurements can only be achieved when the pulse is transmitted and received with vertical polarization. The operating scan modes, which effect the Nyquist velocity interval of the Doppler velocity measurements, together with a short description of the DLR bistatic multiple-Doppler radar network, are given in section 2 . In section 3 , the main steps of the 4DD dealiasing scheme devised by James and Houze (2001) are briefly recalled, and the applied modifications to dealias bistatically measured Doppler velocities are explained. These modifications include 1) processing temporal irregular data with in homogeneous data structures, 2) processing bistatically measured Doppler velocities, and 3) developing an internal dealiasing algorithm to detect and correct those isolated gates which fail the 4DD dealiasing scheme. In section 4, we present results of the modified 4DD scheme and simultaneous measurements of microphysical and dynamical parameters for a downburst-producing thunderstorm moving through southern Germany.

\section{Description of DLR's bistatic multiple-Doppler radar network operations}

Figure 1 illustrates the location of POLDIRAD (also referred as receiver $\mathrm{OP}$ in the following text) and the

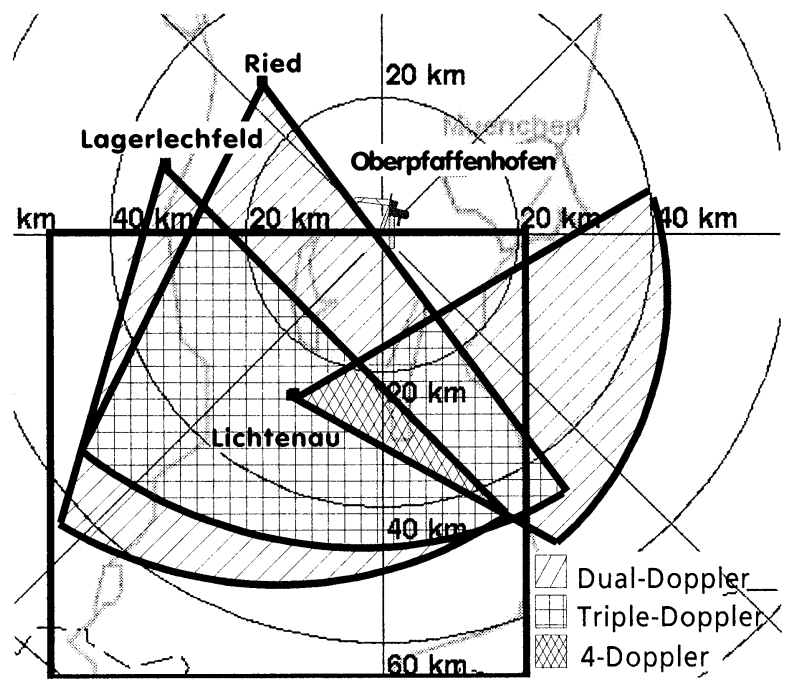

FIG. 1. Map of the bistatic multiple-Doppler radar network at the DLR in Oberpfaffenhofen southwest of Munich in southern Germany consisting of POLDIRAD at Oberpfaffenhofen and three bistatic receivers located at Lichtenau, Lagerlechfeld, and Ried. The investigation areas for multiple-Doppler applications (hatchings) are restricted by the the maximum range and the horizontal antenna aperture of the bistatic antennas. The black box indicates the target area for the downburst-producing thunderstorm event on 9 Jul 2002 (cf. Figs. 7-9).

three bistatic receivers together with the antenna's aperture angles. The target area, indicated schematically, is limited by the maximum range and the power pattern received by the bistatic antenna, which has a horizontal angular aperture covering about $60^{\circ}$. Receiver systems at both Lagerlechfeld and Lichtenau are equipped with antennas having a vertical angular aperture covering $1^{\circ}$ $23^{\circ}$ (corresponding to a maximum height of about 17 $\mathrm{km}$ at a range of $40 \mathrm{~km}$ ). At Ried, a singular antenna having a vertical aperture of $8^{\circ}$ has been installed. Measurements of up to a height of about $6 \mathrm{~km}$ can be taken from a distance of $40 \mathrm{~km}$.

As a result of the transmitter-receiver separation within a bistatic radar system, radar characteristics such as resolution volume length and scattering characteristic both depend on the scattering angle, $\gamma$, spanning the scattering plane between the incident and the scattered ray. For more information concerning bistatic radar characteristics, see Wurman et al. (1993), de Elia and Zawadzki (2000), Friedrich et al. (2000), Takaya and Nakazato (2002), Satoh and Wurman (2003), and references therein.

Bistatic receivers can detect energy scattered in all directions-forward, sideward, and backward $\left(0^{\circ} \leq \gamma\right.$ $\leq 180^{\circ}$ ) - whereas the monostatic receiver is capable of measuring solely the backward-scattered energy and is an exception to the bistatic version where $\gamma=0$. Within the bistatic radar system, surfaces of constant time delay between transmitted and received radar pulses are ellipsoids, with transmitter and receiver at the foci. In the monostatic case, where $\gamma=0$, the surfaces of constant 


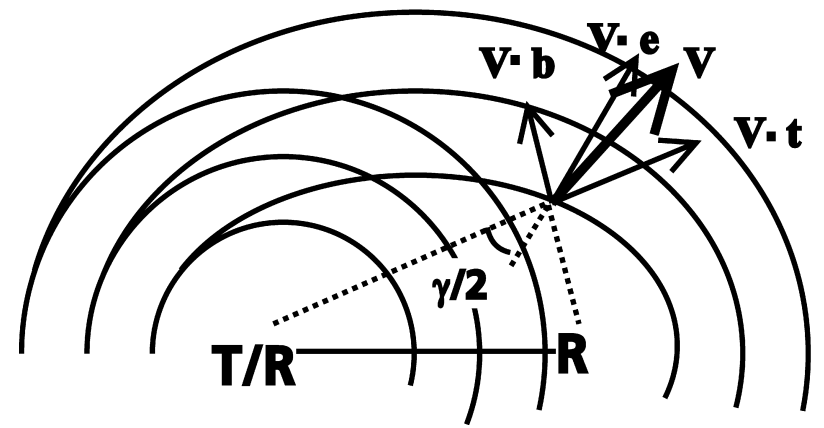

FIG. 2. The decomposition of the wind velocity $\mathbf{V}$ in a bistatic Doppler radar system, with the unit vectors $\mathbf{t}$, e, and $\mathbf{b}$ pointing in the radial direction away from the monostatic receiver, perpendicular to the ellipsoid, and in the radial direction away from the bistatic receiver, respectively. The two-dimensional cross section is obtained along the scattering plane with the scattering angle $\gamma$. The monostatic transmitting-receiving radar is denoted as T/R, the bistatic receiver as $\mathrm{R}$.

delay are spheres centered in the monostatic radar system, as illustrated in Fig. 2. In the case of monostatic radar, only those motions perpendicular to the spheres of constant delay can be observed (Doviak and Zrnić 1984, p. 35); whereas using bistatic radar systems, however, these motions have to be perpendicular to the ellipsoids of constant delay, as illustrated in Fig. 2 (Protat and Zawadzki 1999). The difference in path length when measured by a bistatic receiver within a certain time interval consists of a displacement in the radial direction designated by the unit vector $\mathbf{t}$, and in the receiver direction denoted by the unit vector $\mathbf{b}$. The measured "apparent" velocity, $v_{a}$, has to be projected onto the direction e, which is the unit vector of the direction perpendicular to the ellipsoid of constant delay (Protat and Zawadzki 1999), leading to

$$
v_{e}=\mathbf{V} \cdot \mathbf{e}=\frac{v_{a}}{\cos (\gamma / 2)},
$$

where

$$
v_{a}=\frac{1}{2} \mathbf{V} \cdot(\mathbf{b}+\mathbf{t})
$$

In a Cartesian-coordinate system, $u, v, w$ are the orthogonal components of the wind vector $\mathbf{V}$, oriented along $x, y, z$ (east, north, upward). The Doppler velocity perpendicular to the ellipsoid of constant delay, $v_{e}$, can be written as

$$
\begin{aligned}
v_{e}= & u \frac{\sin \left(\phi_{b}\right) \cos \left(\theta_{b}\right)+\sin \left(\phi_{t}\right) \cos \left(\theta_{t}\right)}{2 \cos (\gamma / 2)} \\
& +v \frac{\cos \left(\phi_{b}\right) \cos \left(\theta_{b}\right)+\cos \left(\phi_{t}\right) \cos \left(\theta_{t}\right)}{2 \cos (\gamma / 2)} \\
& +\left(w-w_{T}\right) \frac{\sin \left(\theta_{b}\right)+\sin \left(\theta_{t}\right)}{2 \cos (\gamma / 2)}
\end{aligned}
$$

with $\phi, \theta$ being the azimuth and elevation angles of the monostatic or bistatic receiver denoted as the subscript $t$ and $b$, respectively. The terminal fall velocity of scattering particles is represented by $w_{T}$.

For monostatic radar systems $\left(\gamma=0, \phi_{b}=\phi_{t}, \theta_{b}=\right.$ $\theta_{t}$ ), Eq. (2) can be simplified and the radial velocity $v_{t}$ can be written as

$$
\begin{aligned}
v_{t}=\mathbf{V} \cdot \mathbf{t}= & u \sin \left(\phi_{t}\right) \cos \left(\theta_{t}\right)+v \cos \left(\phi_{t}\right) \cos \left(\theta_{t}\right) \\
& +\left(w-w_{T}\right) \sin \left(\theta_{t}\right) .
\end{aligned}
$$

For pulsed Doppler radar systems, velocity measurements are unambiguous only insofar as they lie within the Nyquist velocity interval. The Nyquist interval for monostatic radar, $v_{\mathrm{nt}}$, is constant, whereas the Nyquist interval for bistatic reception, $v_{\text {ne }}$ depends on $\gamma$ [cf. Eq. (1)]. Since $v_{\mathrm{ne}} \geq v_{\mathrm{nt}}$, bistatic Doppler velocities are aliased less frequently. As a result, monostatic and bistatic receivers measure a different wind velocity component and have, additionally, different Nyquist velocity intervals. Alternatively, instead of dealiasing $v_{e}$ having a variable Nyquist velocity, the apparent velocity $v_{a}$, which has a constant Nyquist interval, can be dealiased and afterward transformed into $v_{e}$.

The received power, and therewith, the signal-tonoise ratio, depend on the scattering characteristics. Investigations on the Rayleigh scattering process have shown that, for the bistatic Doppler radar system, both the transmitted electromagnetic wave and the receiving antenna should be polarized in a vertical direction (Wurman et al. 1993; de Elia 2000).

When the polarimetric C-band Doppler radar system is equipped with three bistatic receivers, we are able to determine microphysical (i.e., classify radar echoes, identify particle types) and dynamical parameters with high temporal and spatial resolutions. In order to get the complete benefit of both the polarimetric and the multiple-Doppler radar system, measurements have to be taken simultaneously, especially during situations with rapidly evolving weather systems.

Therefore, volumes are scanned using two operating modes, listed in Table 1: 1) For measurements within slowly evolving systems (e.g., frontal passage with stratiform precipitation), a volume is scanned using vertical polarization alone to determine the wind field, followed by a complete volume scan using alternating horizontal and vertical polarization in order to determine microphysical parameters (Hoeller et al. 1994). The update rate is about 10-15 min for each of the two separate volume scans. The sampling frequency is $1200 \mathrm{~Hz}\left(T_{s}\right.$ $=834 \mu \mathrm{s}$ ) leading to a Nyquist velocity interval of $\pm 16.35 \mathrm{~m} \mathrm{~s}^{-1}$. The operating mode is denoted as intensive-scan mode. 2) For rapid evolving systems (e.g., convective systems), only one volume with a high update rate is scanned using alternating horizontal and vertical polarization. The bistatic receivers process only vertically polarized pulses. Therefore, microphysical parameters are sampled at a frequency of $1200 \mathrm{~Hz}$, while the sampling of the dynamical parameters $u$, $v$, using 


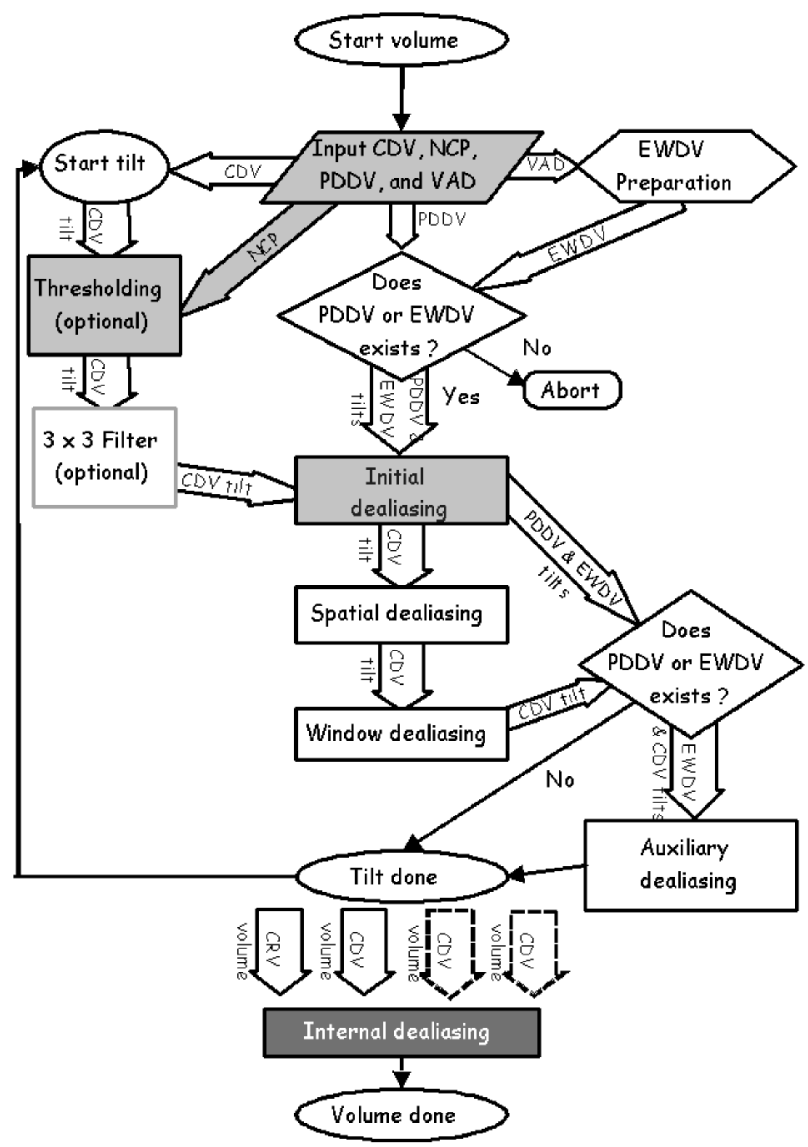

FIG. 3. Flowchart depicting the processing chain for modified 4DD and internal dealiasing (modified from Fig. 1 in James and Houze 2001). Modifications from the original 4DD are marked by gray boxes. Filled, light gray boxes indicate algorithms that were adjusted to bistatically measured Doppler velocity. The internal dealiasing (filled, dark gray box) was added to 4DD completely. Auxiliary dealiasing and the $3 \times 3$ filter illustrated as light gray boxes were not used.

only every second pulse, bisects to $600 \mathrm{~Hz}\left(T_{s}=1667\right.$ $\mu \mathrm{s})$. There is a high demand for the dealiasing scheme because in this case the Nyquist velocity is reduced to $\pm 8.2 \mathrm{~m} \mathrm{~s}^{-1}$.

\section{Dealiasing concept}

\section{a. General remarks}

Each measured Doppler velocity volume was dealiased separately. While the original 4DD scheme was used to dealias radial Doppler velocities (a detailed description of 4DD is given by James and Houze 2001), algorithms of the 4DD scheme were modified in order to dealias Doppler velocities measured by bistatic receivers. Following James and Houze (2001), main steps of the modified 4DD are discussed for bistatically measured Doppler velocities in the following sections to give the reader a short overview. The processing chain performance for bistatically measured Doppler velocities is illustrated in Fig. 3 according to Fig. 1 in James and Houze (2001). While current radial velocity (denoted as CVR in Fig. 1 in James and Houze 2001) and reflectivity field (denoted as DZ in Fig. 1 in James and Houze 2001) sampled by monostatic radar are necessary for the 4DD scheme, modified 4DD uses bistatically measured current Doppler velocity (CDV) and normalized coherent power $(\mathrm{NCP}) .{ }^{1}$

\section{b. Thresholding and filtering}

To be assured of an accurate automated application, all sources of possible error that may lead to an algorithm failure have to be reduced. The first step is to remove all noisy radar data in order to increase the speed and efficiency of the dealiasing algorithm (cf. Fig. 3, thresholding). In the monostatic signal processor, data are considered for further data processing only if the measured power exceeds a value of about $-108 \mathrm{dBm}$. Since there is currently no information available on either NCP or spectral width from the bistatic receiver located at the transmitting site, reflectivity measured at the monostatic receiver is used as a threshold to remove noisy data. For the velocities measured by remote bistatic receivers where NCP is recorded, NCP must be larger than 0.3 to be considered for processing.

In the second step of the original 4DD scheme, isolated gates are eliminated by using a Bergen and Albers (1988) filter in order to avoid dealiasing failure (cf. Fig. $3,3 \times 3$ filter). Test runs with the DLR dataset showed that this filter increases the number of missing values in areas surrounded by good-values areas. Therefore, the Bergen-Albers filter is not applied here.

\section{c. Initial dealiasing}

The initial dealiasing concept by James and Houze (2001) using the vertical dimension along with the time dimension in order to constrain the initial dealiasing is applied to the monostatic and bistatic datasets in its entirety. In the first step of the internal dealiasing algorithm a three-dimensional smoothed, synthetic wind field (EWDV) is derived from a velocity-azimuth display (VAD) analysis (Lhermitte and Atlas 1961; Browning and Wexler 1968) or a sounding (cf. Fig. 3, EWDV Preparation). Alternatively, the wind information of the previously dealiased Doppler velocity scan (PDDV) is used for initial dealiasing. While a VAD or sounding is used for the first time step to be dealiased, successive scans are dealiased using a previously dealiased scan when the time difference between the two scans is less than $20 \mathrm{~min}$. This EWDV is compared to the velocities still to be dealiased within the initial dealiasing algo-

\footnotetext{
${ }^{1}$ Index related inversely to the spectral width ranging from 0 to 1. At the bistatic receivers it is calculated as $\mathrm{NCP}=\left|R_{1}\right| / R_{0}$ with $R_{0}, R_{1}$ being the 0 th and $1 \mathrm{st}$ moment of the autocorrelation function taken from the Doppler power spectrum (for more details, see Friedrich 2002, p. 114).
} 
rithm (cf. Fig. 3, initial dealiasing). They are considered only as not aliased when the velocity difference of the same gate is less than $0.25 v_{n}$, where $v_{n}$ is the Nyquist velocity interval. In addition, the difference to the nearest gate in the previous tilt above has also to be less than $0.25 v_{n}$. Dealiased gates are hereafter denoted as "good" meaning that they do not require any further treatment.

The following modifications are applied to the original 4DD scheme in order to deal with bistatically measured data. To begin with, data measured by bistatic receivers are interpolated onto a spherical coordinate system centered around the transmitting radar, so that both monostatically and bistatically measured datasets can be considered by the 4DD scheme. Second, EWDV used as a reference field is not only projected onto the radial velocity component for the monostatic measurements [Eq. (3)] but also onto the velocity component measured by each bistatic receiver according to Eq. (2). Third, since the Nyquist velocity remains constant in this case, Doppler velocities measured by bistatic receivers are dealiased using the apparent velocity component. Note that each receiver measures a different component of the wind vector (cf. section 2) and that the threshold of the velocity difference is set at $0.25 v_{n}$ for both applications.

The 4DD code was developed to continuously scan volume data, and it therefore assumes that the first and last rays are adjacent to each other. It also assumes that, for each ray within a given sweep, there exists a ray above it, excepting the highest sweep. Further this assumption is that all volumes are considered to have the same spatial structure. These conditions cannot be fulfilled by the DLR datasets due to the fact that the sector scanning operation and recording of rays does not occur at fixed azimuth and elevation angles. Therefore, to cope with the inhomogeneity of data the algorithm was modified in a way that sweeps were not assumed to scan primarily $360^{\circ}$. For instance, a procedure was added to the initial dealiasing algorithm in order to search for the nearest ray in the previous sweep because 4DD assumed it to have the same index.

\section{d. Spatial, window, and auxiliary dealiasing}

Spatial, window, and auxiliary dealiasings are completely adopted from James and Houze (2001) and will be explained only shortly in the following section (cf. Fig. 3). Using the good gates determined so far, spatial continuity within a sweep is used to dealias other gates when the difference in velocity between two adjacent gates is less than a user-defined threshold (default $\left.0.4 v_{n}\right)$. Each gate surrounded by a good gate is dealiased by an integer so that the velocity difference between each neighboring good gate is less than $0.4 v_{n}$. Spatial dealiasing starts scanning outward along each radial and progresses radial by radial in a clockwise direction. During each successive pass, 4DD alternates between clockwise and counterclockwise progression while continuing to scan radially outward. At the third pass the threshold is set to $v_{n}$, and each gate must agree only with the majority of the adjusted good gates. Dealiasing is continued until completing a total of 10 passes.

Some gates may remain that are not directly adjacent to good gates. Throughout the sweep, a window of dimensions $11 \times 11$ is applied to the remaining gates that computes the number of good gates within the window and the standard deviation of the speed. If the number of good gates is sufficient (default 5) and the standard deviation small enough (default less than $\left.0.8 v_{n}\right)$, the speed of the central gate is adjusted modulo $2 v_{n}$, otherwise the window dimensions is expanded to $21 \times 21$.

Eventually, auxiliary dealiasing requires both VAD and previously dealiased scans. When there is a small enough (default threshold relaxed to $0.49 v_{n}$ ) speed difference between the gate under consideration and the corresponding one in the VAD or previous scan, remaining gates are set as good.

\section{e. Internal dealiasing}

So far each Doppler velocity component passed the 4DD scheme separately. After passing the 4DD scheme, the dealiasing status - that is, bad/missing, dealiased, or still aliased-together with the reliability (expressed by the routine used for dealiasing) of each Doppler velocity is recorded. Measuring several individual wind components simultaneously enables us now to merge this information together with the wind information itself and thereby detect and dealias gates which have failed the dealiasing process so far (cf. Fig. 3, internal dealiasing). Internal dealiasing can be applied to those observation areas within the DLR's bistatic multipleDoppler radar network where equations used to determine a horizontal wind are overdetermined (see triple and quadruple Doppler areas in Fig. 1). The aim is to determine the horizontal wind vector using two dealiased velocity components and afterward calculate a velocity component of a receiver not involved in the dual-Doppler analysis (for more information on wind synthesis using monostatic and bistatic velocity components, see Friedrich and Hagen 2004). This recalculated velocity component is then compared to the measured and still aliased velocity component. Since each receiver measures a different component of the wind vector, it is most likely that only one Doppler velocity component is aliased, so that the internal dealiasing algorithm can already be applied for a combination of three receivers. 
According to Eq. (2) disregarding $w$, the apparent velocity measured by the receiver $i$ can be expressed as

$$
v_{a}(i)=a_{i} u+b_{i} v
$$

where

$$
\begin{aligned}
a_{i} & =\frac{\sin \phi_{b_{i}} \cos \theta_{b_{i}}+\sin \phi_{t} \cos \theta_{t}}{2}, \\
b_{i} & =\frac{\cos \phi_{b_{i}} \cos \theta_{b_{i}}+\cos \phi_{t} \cos \theta_{t}}{2} .
\end{aligned}
$$

In the monostatic case, the equation is reduced according to Eq. (3) and $v_{a}(i)$ becomes $v_{t}$. The horizontal wind vector using two Doppler velocity components, $v_{a}(i)$, $v_{a}(j)$, measured by receivers $i$ and $j$ can be determined exactly as

$$
\begin{aligned}
& u_{i j}=\frac{b_{j} v_{a}(i)-b_{i} v_{a}(j)}{\Delta i j}, \\
& v_{i j}=\frac{a_{i} v_{a}(j)-a_{j} v_{a}(i)}{\Delta_{i j}},
\end{aligned}
$$

where $\Delta_{i j}=a_{i} b_{j}-a_{j} b_{i}$. The velocity component of a third receiver, $k$, one not involved in the dual-Doppler analysis [Eqs. (5), (6)], can be calculated from the horizontal wind components $u_{i j}$ and $v_{i j}$ as

$$
\begin{aligned}
v_{a}^{i j}(k) & =a_{k} u_{i j}+b_{k} v_{i j}, \\
& =\frac{a_{k} b_{j}-a_{j} b_{k}}{\Delta_{i j}} v_{a}(i)+\frac{a_{i} b_{k}-a_{k} b_{i}}{\Delta_{i j}} v_{a}(j), \\
& =\frac{\Delta_{k j}}{\Delta_{i j}} v_{a}(i)+\frac{\Delta_{i k}}{\Delta_{i j}} v_{a}(j) .
\end{aligned}
$$

Afterward, $\boldsymbol{v}_{a}^{i j}(k)$ is compared to the measured component of receiver $k$. Assuming the margin for error, $\epsilon$, to be the same for each of the three receivers $\{$ that is $\epsilon=\delta\left[v_{a}(i)\right]=\delta\left[v_{a}(j)=\delta\left[v_{a}(k)\right]\right\}$, the margin for error between $v_{a}^{i j}(k)$ and the measured component of receiver $k$ then becomes

$$
\begin{aligned}
\delta & {\left[v_{a}(k)-v_{a}^{i j}(k)\right] } \\
& =\delta\left[v_{a}(k)-\frac{\Delta_{k j}}{\Delta_{i j}} v_{a}(i)-\frac{\Delta_{i k}}{\Delta_{i j}} v_{a}(j)\right] \\
& =\delta\left[v_{a}(k)\right]+\delta\left[\frac{\Delta_{k j}}{\Delta_{i j}} v_{a}(i)\right]+\delta\left[\frac{\Delta_{i k}}{\Delta_{i j}} v_{a}(j)\right] \\
& =\epsilon+\left|\frac{\Delta_{k j}}{\Delta_{i j}}\right| \epsilon+\left|\frac{\Delta_{i k}}{\Delta_{i j}}\right| \epsilon \\
& =\frac{\left|\Delta_{i j}\right|+\left|\Delta_{k j}\right|+\left|\Delta_{i k}\right|}{\left|\Delta_{i j}\right|} \epsilon .
\end{aligned}
$$

The difference between $v_{a}(k)$ and $v_{a}^{i j}(k)$ must be of the same order of magnitude as the margin for error in measuring, shown as (a)

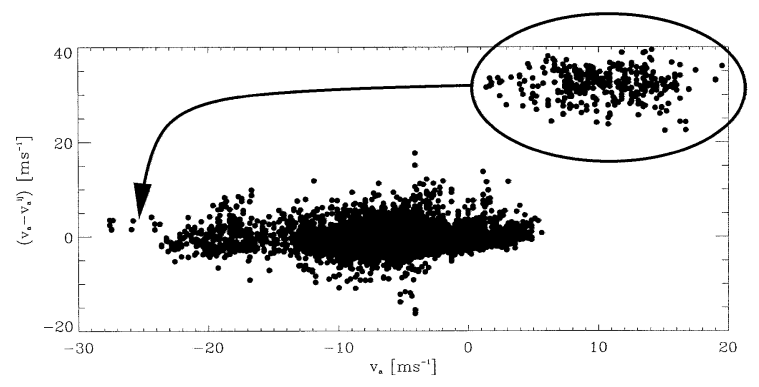

(b)

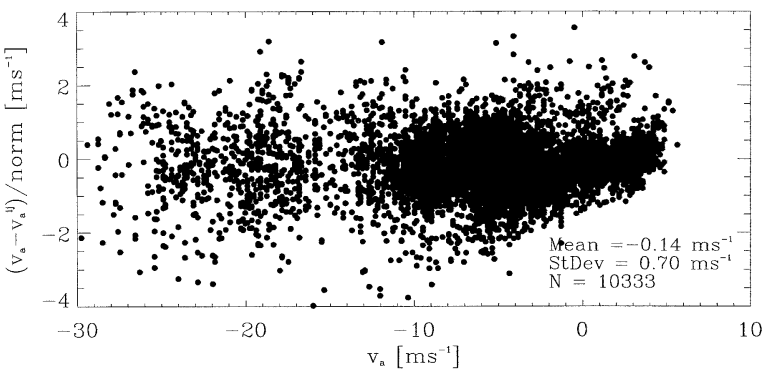

FIG. 4. Internal dealiasing of dealiased, isolated Doppler velocities measured by receiver Lichtenau (denoted as $v_{a}$ ) during a thunderstorm event at 1556 UTC 9 Jul 2002. The dealiasing bases on the wind information from receivers POLDIRAD and Ried (denoted as $v_{a}^{i j}$ ). (a) The algorithm detects dealiased gates due to large velocity differences between $v_{a}$ and $v_{a}^{i j}$ (marked by the ellipse) and dealiases those data into the respective Nyquist velocity intervals as indicated by the arrow. (b) After the dealiasing of single gates, the differences are normalized to the measurement geometry. Mean value (Mean), standard deviation (StDev), and number of samples $(\mathrm{N})$ are denoted.

$$
\left|v_{a}(k)-v_{a}^{i j}(k)\right| \leq \frac{\left|\Delta_{i j}\right|+\left|\Delta_{k j}\right|+\left|\Delta_{i k}\right|}{\left|\Delta_{i j}\right|} \epsilon,
$$

otherwise either the measured data will be contaminated or gates will not be correctly dealiased. Figure 4 portrays the internal dealiasing of Doppler velocities measured by receiver Lichtenau for a downburst-producing thunderstorm which took place at 1556 UTC on 9 July 2002. Wind information determined from measurements of receivers POLDIRAD and Ried are utilized as the nonaliased reference field. Erroneous Doppler velocities (marked by a circle in Fig. 4a) are indicated by great differences of $\left(v_{a}-v_{a}^{i j}\right)$ ranging between 20 and $40 \mathrm{~m}$ $\mathrm{s}^{-1}$. These points can therefore correspond to those remaining dealiased gates which fail the dealiasing algorithm so far but which can then be dealiased by trying combinations of velocities modulo the Nyquist velocity until the difference between the $v_{a}^{i j}$ and $v_{a}$ Lichtenau is smaller than the empirical chosen threshold of $6 \mathrm{~m} \mathrm{~s}^{-1}$. The aliased velocities are dealiased into the respective velocity, as illustrated by the arrow in Fig. 4a. In order to assess the quality of the measurement, Doppler velocities are normalized by the scattering angle with norm $=\left(\left|\Delta_{i j}\right|+\left|\Delta_{k j}\right|+\left|\Delta_{i k}\right|\right) /\left(\left|\Delta_{i j}\right|\right)$. Normalized Doppler 
(a)

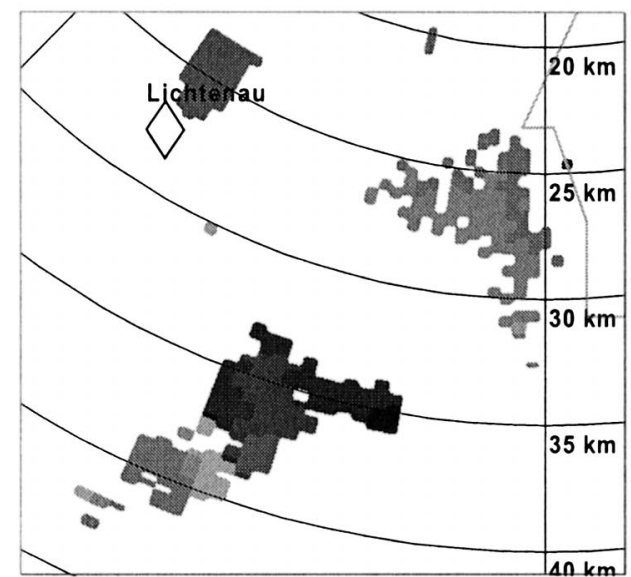

(b)

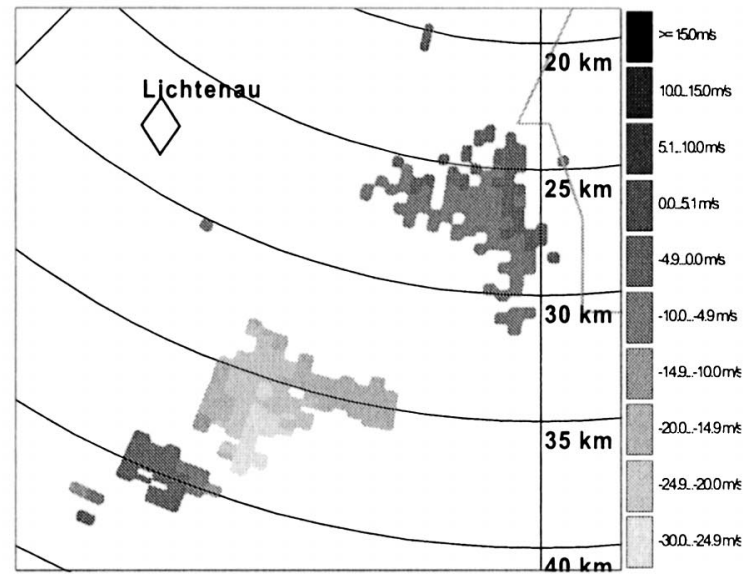

FIG. 5. (a) Doppler velocities measured by receiver Lichtenau at $11^{\circ}$ showing that the isolated cell at $35-40-\mathrm{km}$ range SSW of OP was not dealiased by the original 4DD. (b) After applying the internal dealiasing algorithm both the isolated cell was dealiased, and noisy data at a range of about $25 \mathrm{~km}$ close to receiver Lichtenau were removed. Data were measured at 1556 UTC 9 Jul 2002. The Doppler velocities that failed 4DD and the results of the internal dealiasing of the isolated cell can also be seen in Fig. 4. Range rings are centered around POLDIRAD.

velocities are illustrated in Fig. $4 \mathrm{~b}$ for the downburstproducing thunderstorm at 1556 UTC 9 July 2002. Figure 5 gives an example of successfully applying the internal dealiasing algorithm for an isolated cell at an elevation of $11^{\circ}$. The Doppler velocities at a range of 34-38 km were not identified as aliased by the 4DD scheme (Fig. 5a). However, with the internal dealiasing algorithm, the aliased Doppler velocities can be detected and dealiased (Figs. 4a, 5b). Furthermore, erroneous data like those illustrated for instance in Fig. 5a at a 24-27-km range can be detected and removed by the internal dealiasing algorithm (cf. Fig. 5b). The differences in measurements within the multiple-Doppler area

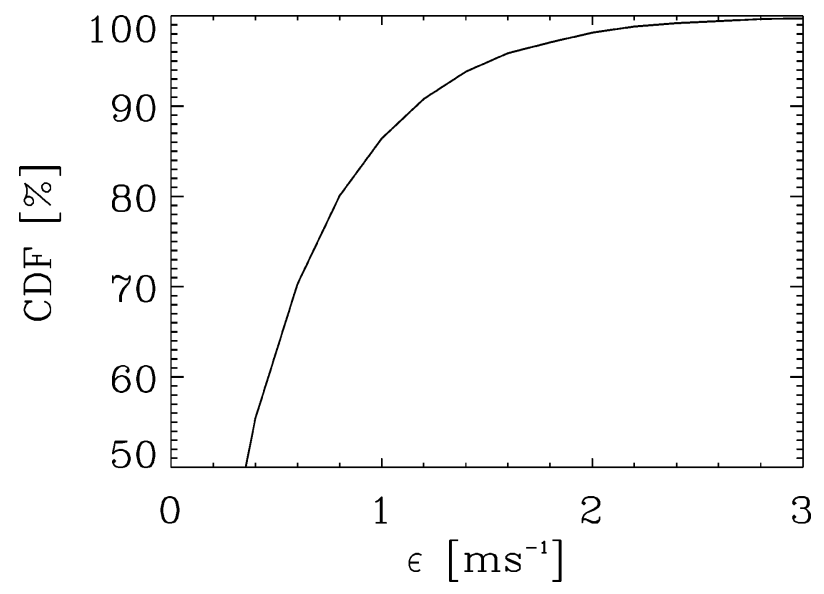

FIG. 6. Quality control of the Doppler velocity measurements and the dealiasing procedure illustrating the empirical cumulative probability distribution function, $\mathrm{CDF}(\%)$, and the measurement error, $\epsilon$, $\left(\mathrm{m} \mathrm{s}^{-1}\right)$. Data were sampled at 1556 UTC $9 \mathrm{Jul}$ 2002. CDF is related to the velocity difference between receiver Lichtenau and the combination of receivers POLDIRAD and Ried. have to be within a velocity interval of $\pm 10 \mathrm{~m} \mathrm{~s}^{-1}$ in order to differentiate these points from aliased points. Finally, the quality of Doppler velocity measurements itself and the dealiasing process can be assessed by calculating the empirical cumulative probability function $(\mathrm{CDF})$, as illustrated in Fig. 6. The figure shows that $99 \%$ of the velocity measurements satisfies Eq. (9) for $\epsilon=2.3 \mathrm{~m} \mathrm{~s}^{-1}$, and $95 \%$ for $\epsilon=1.5 \mathrm{~m} \mathrm{~s}^{-1}$, both of which lie within the scale for bistatically measured Doppler velocity. This procedure is also used for quality-control purposes within an automated evaluation of bistatically measured wind field (Friedrich and Hagen 2003).

\section{Scan mode and algorithm performance}

Scan mode and algorithm performance of the modified 4DD scheme are exemplified for a downburst-producing thunderstorm event which took place on the afternoon of 9 July 2002 during the Vertical Exchange and Orography measuring campaign (VERTIKATOR). The VERTIKATOR project aims at improved understanding of how shallow and deep convection over hilly and mountainous terrain get initiated and develop. A particular focus was investigating the interaction between synoptic-scale settings with local effects such as the heat low over mountain ranges or valley flows with respect to convective transport. Wind velocity measurements were achieved using POLDIRAD and the bistatic receivers at Lichtenau and Ried (Fig. 1).

A depression centered to the north of Ireland entailed a cyclonic flux in the western part of Europe. The storm developed in the warm-sector air mass ahead of a cold front crossing Europe. At 1200 UTC, convection developed within the northern Alps. Initial convective 
TABLE 1. Configurations of the operating modes in order to derive microphysical information determined by reflectivity factor ( $Z$ ), differential reflectivity $\left(Z_{\mathrm{DR}}\right)$, and linear depolarization ratio (LDR) as well as dynamical parameters $u$ and $v$. Transmitted (denoted as Tx) and received polarization (denoted as $\mathrm{Rx}$ ) can be either horizontal (denoted as $\mathrm{H}$ ) or vertical (denoted as V).

\begin{tabular}{|c|c|c|c|c|c|c|c|}
\hline & $\mathrm{PRF}(\mathrm{Hz})$ & $v_{n}\left(\mathrm{~m} \mathrm{~s}^{-1}\right)$ & Update (min) & Parameters & $\mathrm{Tx}$ & Mono. Rx & Bist. Rx \\
\hline Intensive-scan mode (Doppler) & 1200 & 16.35 & $10-15$ & $u, v$ & $\mathrm{~V}$ & $\mathrm{~V}$ & V \\
\hline Intensive-scan mode (dual Pol) & 1200 & 16.35 & $10-15$ & $Z, \mathrm{LDR}, Z_{\mathrm{DR}}$ & $\mathrm{H}, \mathrm{V}$ & $\mathrm{H}, \mathrm{V}$ & - \\
\hline Rapid-scan mode & $\begin{array}{r}1200 \\
600\end{array}$ & $\begin{array}{c}16.35 \\
8.2\end{array}$ & 5 & $Z$, LDR, $Z_{\mathrm{DR}}$ & $\mathrm{H}, \mathrm{V}$ & $\mathrm{H}, \mathrm{V}$ & $\bar{V}$ \\
\hline
\end{tabular}

cells were released few hours later traveling northeastward and passing the investigation area at about 1500 UTC. This phenomenon was observed until 1800 UTC. At that time, single mesoscale convective cells merged into a mesoscale convective system.

Data were sampled between 1400 and 2030 UTC in both the intensive and rapid-scan mode (cf. Table 1). At the start, each mode alternated to ensure that the time difference between the two volume scans was about 5$10 \mathrm{~min}$. This strategy was set up in order to better analyze both scan modes and test the comprehensiveness of the modified 4DD scheme when the Nyquist velocity interval reduced from \pm 16.35 to $\pm 8.2 \mathrm{~m} \mathrm{~s}^{-1}$. In addition to the volume scans, VAD scans at an elevation of $20^{\circ}$ were determined in order to observe the vertical wind profile of the horizontal wind which was used as initial wind information for the 4DD scheme.

Automated dealiasing started with the volume scan recorded at 1400 UTC. The VAD analysis was used to derive the three-dimensional environmental wind field for the initial dealiasing algorithm. The successive volume scans used the wind information from the previously dealiased volume scan.

\section{a. Intensive-scan mode}

In the intensive-scan mode, the electromagnetic wave is transmitted vertically polarized and only vertical polarization is received (cf. Table 1). Figures $7 \mathrm{a}$ and $8 \mathrm{a}$ illustrate Doppler velocity fields measured when using the intensive-scan mode by receivers POLDIRAD and Ried at 1556 UTC, respectively. Doppler velocities were sampled at a PRF of $1200 \mathrm{~Hz}$ corresponding to a Nyquist velocity interval of $\pm 16.35 \mathrm{~m} \mathrm{~s}^{-1}$. Velocity aliasing occurs in the area of the main convective cells south (S) of OP at about a 50-km range, south-southwest (SSW) of OP at a 30-50-km range, and west-southwest (WSW) of OP at a $40-50-\mathrm{km}$ range with reflectivity values larger than $40 \mathrm{dBZ}$. Figure 9a exhibits the reflectivity field at 1602 UTC at $6.5^{\circ}$ elevation. In Figs. 7a and 8a, the aliased Doppler velocities stand out clearly as dark areas, and velocity values change their signs ranging from $-16.35 \mathrm{~m} \mathrm{~s}^{-1}$ in the dealiased areas to $10-16 \mathrm{~m} \mathrm{~s}^{-1}$ in the aliased areas. Figures $7 \mathrm{~b}$ and $8 \mathrm{~b}$ both portray the dealiased Doppler velocities using the modified 4DD scheme. The velocities range between -16.35 to $-35 \mathrm{~m} \mathrm{~s}^{-1}$ within the previously aliased areas. Note that in the modified 4DD scheme, in order to remove noisy data, the reflectivity threshold was applied to the radial velocity field (cf. Figs. 7a,b) as explained in section $2 \mathrm{~b}$. The 4DD dealiasing scheme successfully dealiased large parts of the Doppler velocity. Isolated dealiased gates at high elevations were detected by the internal dealiasing algorithm, which must afterwards be corrected (cf. section 2e; Fig. 4), so that the complete volume can be successfully dealiased. Figures $4 \mathrm{a}$ and 5 give an example of applying the internal dealiasing algorithm to isolated cells. The isolated areas were not dealiased by the modified 4DD scheme but could be clearly identified as aliased areas by the internal dealiasing algorithm (cf. Fig. 4a). Note that even with the naked eye, this area could not be identified as an aliased region.

\section{b. Rapid-scan mode}

When scanning in the rapid mode, the transmitted polarization alternates between horizontal and vertical. Bistatic receivers, on the other hand, evaluate only those pulses having a vertical polarization (see Table 1). To demonstrate the utilization of the modified 4DD scheme, the rapid-scan mode was set to about 6 min later than the intensive-scan mode (cf. section 4a). Note that in the rapid-scan mode it is possible to simultaneous measure both dynamical and microphysical properties.

Figures $7 \mathrm{c}$ and $8 \mathrm{c}$ illustrate the Doppler velocity field measured by receivers POLDIRAD and Ried at 1602 UTC, respectively. Owing to a velocity sampling of PRF $=600 \mathrm{~Hz}$, the Nyquist velocity interval was thence reduced to $\pm 8.2 \mathrm{~m} \mathrm{~s}^{-1}$ (cf. Table 1). In this instance, Doppler velocities were aliased several times almost within the entire observation area-for example, the area being at a range of $20-50 \mathrm{~km}$ and at an elevation of $11^{\circ}$ (cf. Figs. 7a,b and 8a,b). Velocity values changed from -8.2 to $8.2 \mathrm{~m} \mathrm{~s}^{-1}$. At a range of $40 \mathrm{~km}$, the Doppler velocities were aliased 4 times. After applying the modified 4DD scheme, the velocity fields were dealiased as exhibited in Figs. 7d and 8d. Again, the Doppler velocities of the previously aliased areas ranged between -10 to $-40 \mathrm{~m} \mathrm{~s}^{-1}$ at an elevation of $11^{\circ}$. Note that at a range of $30-50 \mathrm{~km}$, the direction of the wind components measured by receivers POLDIRAD and Ried varied only by about $20^{\circ}-30^{\circ}$.

Dealiased wind fields using the rapid-scan mode (cf. Figs. $7 b, 8 b$ ) were consistent with those using the in- 
(a)

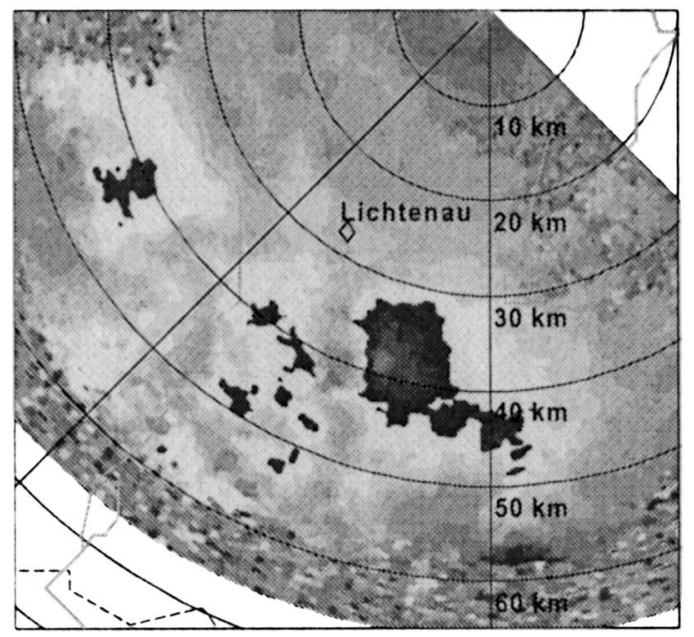

(c)

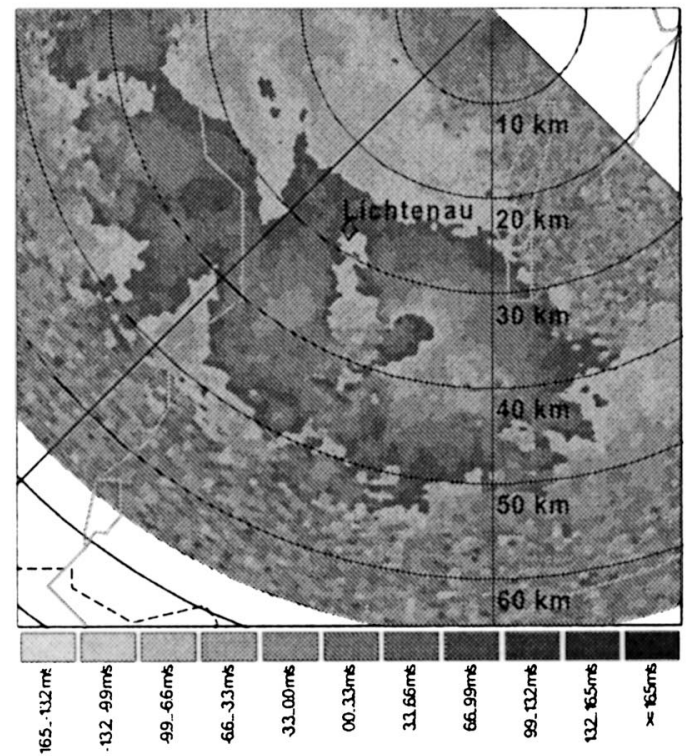

(b)

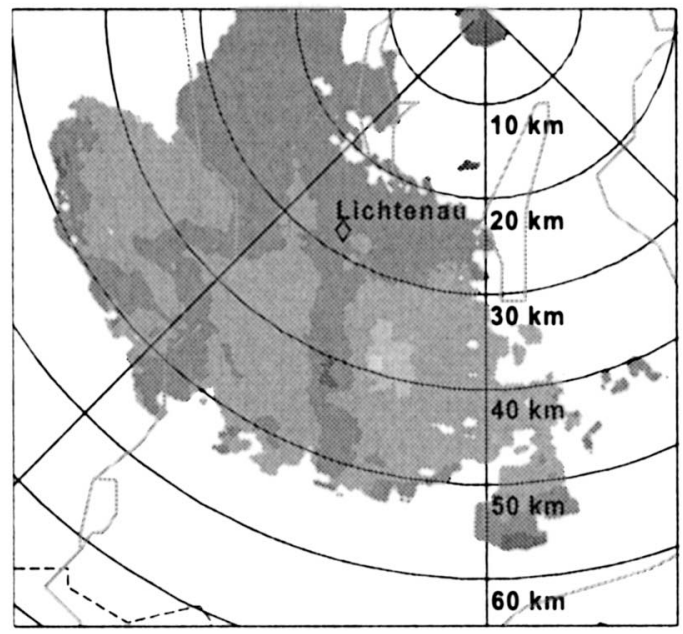

(d)

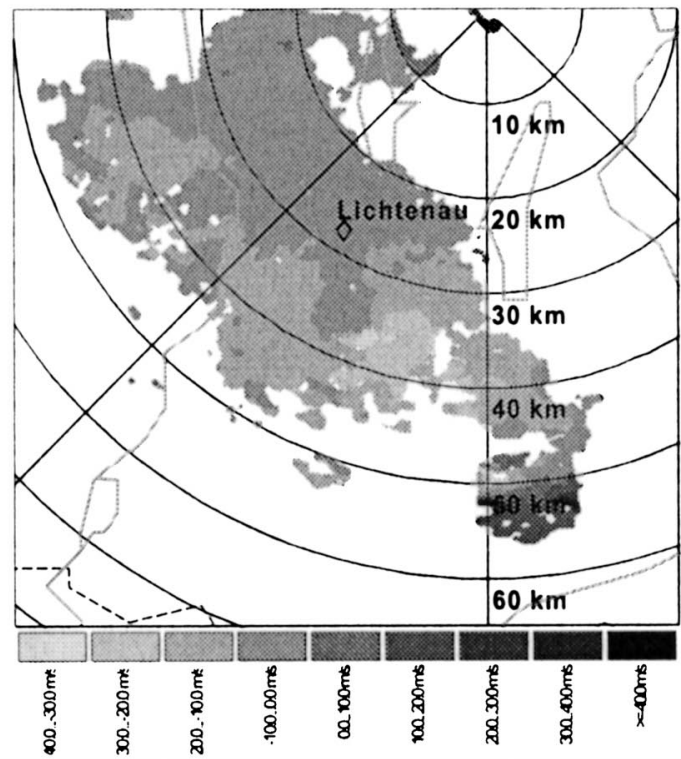

FIG. 7. Doppler velocity measured by POLDIRAD at an elevation angle of $11^{\circ}$ on $9 \mathrm{Jul} 2002$. Velocities were sampled either at a PRF of $1200 \mathrm{~Hz}$ (intensive-scan mode at $1556 \mathrm{UTC}$ ) illustrated in the top panels or at PRF $=600 \mathrm{~Hz}$ (rapidscan mode at 1602 UTC) portrayed in the lower panels. Based on a Nyquist velocity of \pm 16.35 and $\pm 8.2 \mathrm{~m} \mathrm{~s}^{-1}$, respectively, the modified 4DD scheme is applied to the raw Doppler velocities shown in (a) and (c). The respective dealiased Doppler velocities are illustrated in (b) and (d), respectively. Range rings are centered around POLDIRAD.

tensive-scan mode (Figs. 7d, 8d), showing that all aliased Doppler velocities were properly dealiased.

During a 6-min time interval between the two volume scans, a convective cell located $\mathrm{S}$ of $\mathrm{OP}$ at about a $50-$ $\mathrm{km}$ range developed. This cell is scarcely discernible in Fig. 7c, but is clearly identified in the reflectivity field having values of about $20-35 \mathrm{dBZ}$ at $11^{\circ}$ elevation (reflectivity field at $6.5^{\circ}$ is shown in Fig. 9a). Unfortu- nately, this area was monitored only by the monostatic radar so that the internal dealiasing algorithm could not be applied. Nevertheless, a visual check renders the dealiased fields plausible. In the next time stage at 1608 UTC, this area was partly covered by receiver Lichtenau to facilitate applying the internal dealiasing algorithm showing that this area was correctly dealiased by 4DD at 1602 and 1608 UTC. To further improve its success, 
(a)

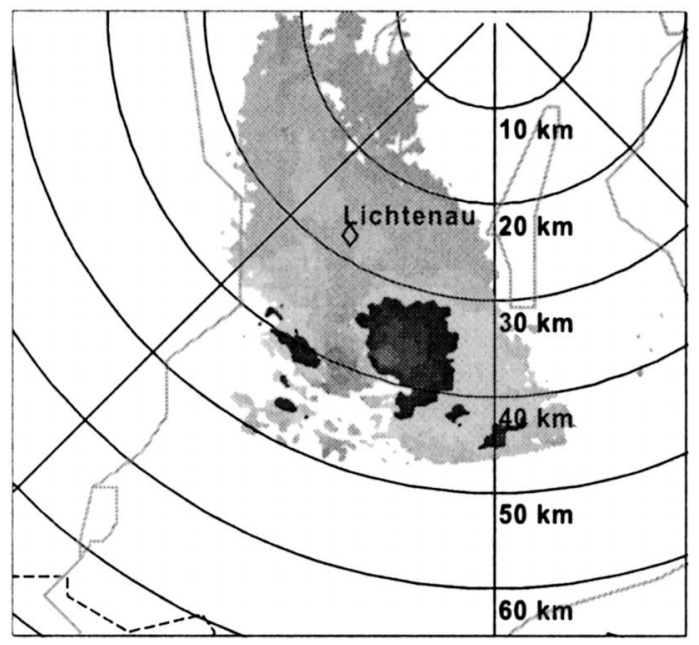

(c)

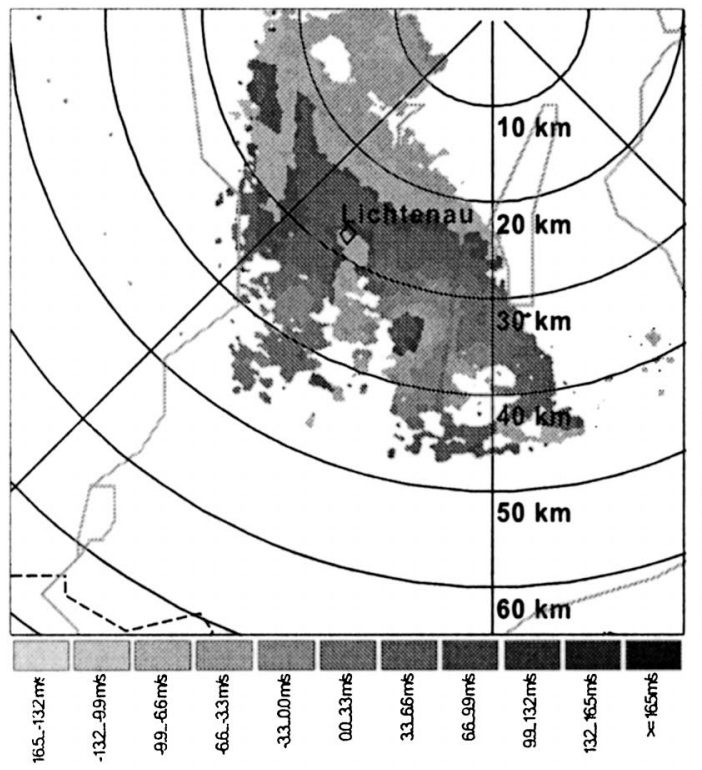

(b)

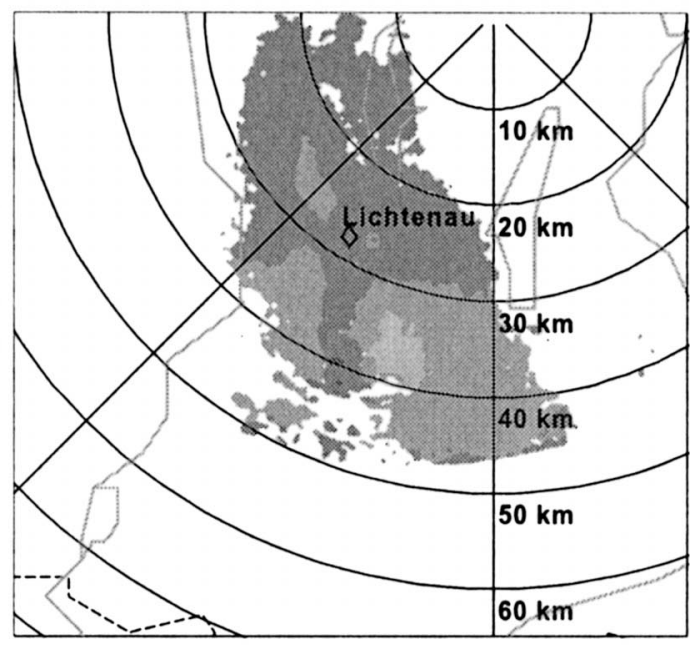

(d)

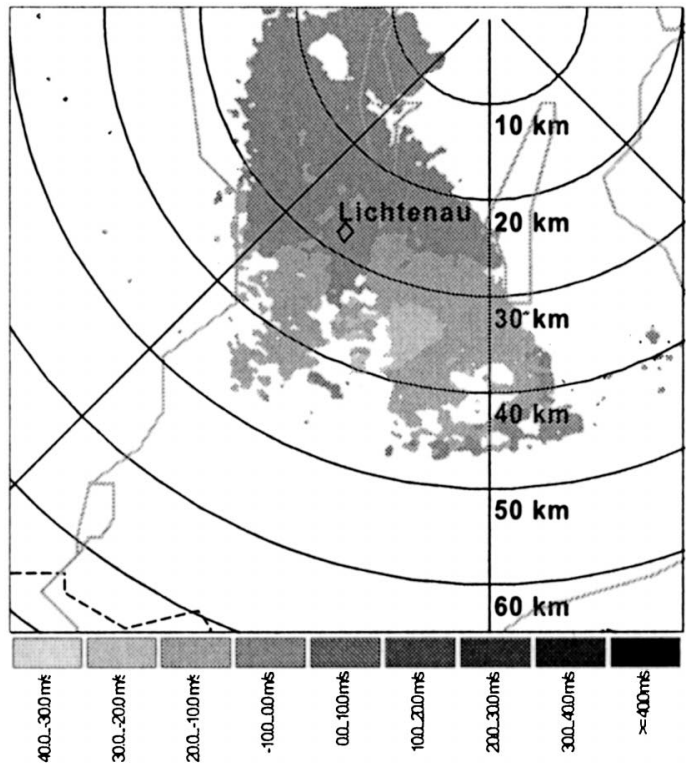

FIG. 8. As in Fig. 7, except that Doppler velocity fields were obtained by receiver Ried.

4DD could be backtracked in time to compare Doppler velocities. One can also apply such a technique to 4DD in a similar way as achieved for the internal dealiasing. In the case of its failing, the volume scan possessing more Doppler velocity information; that is, the scan with a higher number of receivers covering this particular area can be used as reference to dealias the other time step. This procedure, however, is probably very time consuming and is therefore not recommended for a realtime application.

Comparing dealiasing results between the intensive and the rapid-scan modes shows that the modified 4DD dealiasing scheme succeeds in this case even for a low Nyquist velocity interval and a weather situation with high wind shear. Even within the small bistatic observation area at low elevations, which is restricted by the receiving antenna pattern, Doppler velocities can be dealiased successfully by modified 4DD. Again, dealiasing isolated gates at high elevations presents problems, but these can be solved for the overdetermined areas by an internal dealiasing algorithm.

Figure 9 exhibits the reflectivity field at $5.3^{\circ}$ elevation and the hydrometeor classification at $9.6^{\circ}$ elevation, both superimposed on the horizontal wind field at the re- 
(a)

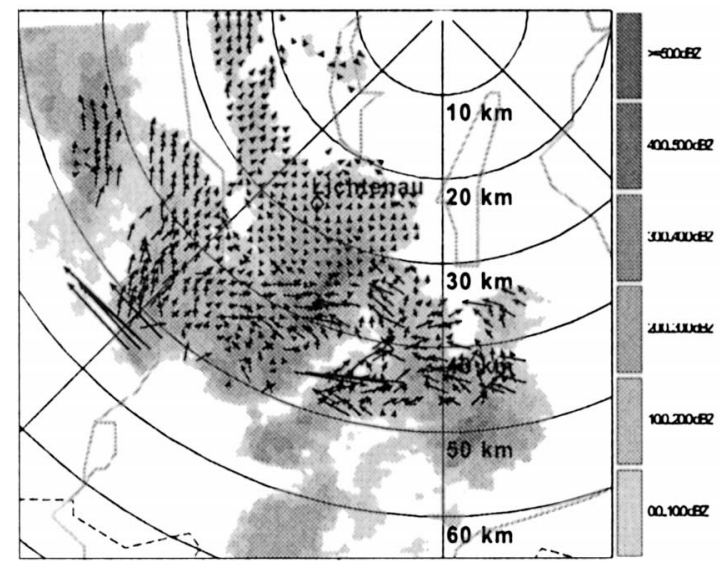

(b)

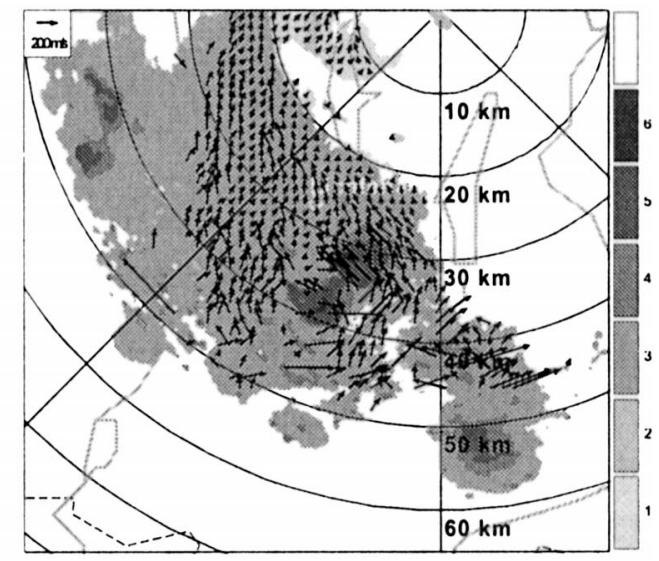

FIG. 9. Combination of simultaneous measurements of the horizontal wind field and microphysical parameters for a downburst-producing thunderstorm on $9 \mathrm{Jul}$ 2002. (a) The reflectivity factor field measured by the monostatic radar in $\mathrm{dBZ}$ at an elevation of $5.3^{\circ}$ and (b) hydrometeor classification after Hoeller et al. (1994) at an elevation of $9.6^{\circ}$ are presented. Both fields are overlaid by the horizontal wind field in $\mathrm{m} \mathrm{s}^{-1}$ [reference vector, top left corner of (b)] determined from measurements of receivers POLDIRAD, Ried, and Lichtenau at the respective elevation angle. Hydrometeor types (grayscale) are denoted as 1) small raindrops below the melting layer; 2) large raindrops; 3) small dry graupel, snow above the melting layer; 4) small wet melting graupel, large dry graupel, small dry hail; 5) dry hail; and 6) wet hail.

spective elevation angle. Thunderstorm cells with values larger than $40 \mathrm{dBZ}$ located WSW, SSW, and S of OP are visible in the reflectivity field. The hydrometeors were classified after Hoeller et al. (1994). As indicated in Fig. 9b, both dry and wet hail as well as graupel were observed within the convective areas, graupel and snow dominated the surrounding area above the melting layer, and there were raindrops below it. Hail stones up to a size of $3 \mathrm{~cm}$ were observed at the ground in the downburst area. The cells themselves moved at a speed of about $8 \mathrm{~m} \mathrm{~s}^{-1}$ northeastward. Highly divergent horizontal wind fields within the thunderstorm cells were observed by the bistatic Doppler radar network (Fig. 9b).

At ground level, the wind vectors showed a high variability in direction and wind speeds of about $\pm 10 \mathrm{~m}$ $\mathrm{s}^{-1}$, whereas at higher levels high wind shear was observed (Fig. 9a). Above a height of about $9 \mathrm{~km}$, the wind outside the thunderstorm core came mainly from a south-southwesterly direction and had values ranging from between 20 to $30 \mathrm{~m} \mathrm{~s}^{-1}$. Within the high reflectivity core of the thunderstorm cell SSW of OP, highly convergent wind fields were observed at $5.3^{\circ}$ at a range of $35-45 \mathrm{~km}$ (which corresponds to a height of $3.5 \mathrm{~km}$ above ground level). The same phenomenon was observed by the thunderstorm cell $\mathrm{S}$ of $\mathrm{OP}$ at $5.3^{\circ}$ and $9.6^{\circ}$. On both occasions, wind field patterns could be ascribed to an early state of microburst development. This assumption was reinforced by a microburst observation at 1730 UTC southwest of Munich that corresponded both to the advection velocity of about $8 \mathrm{~m}$ $\mathrm{s}^{-1}$ (which is about $90 \mathrm{~min}$ for $50 \mathrm{~km}$ ) and its direction (northwest).

On that particular day, a high downburst potential was also reproduced by the evolution of the vertical temperature profile measured by the Munich sounding at 1200 and 1800 UTC. Up until 1800 UTC, a greater than $300-\mathrm{hPa}$-deep layer with a steep laps rate and increasingly dry air near the ground had formed, enabling vigorous downdrafts from thunderstorm to develop.

\section{Summary and conclusions}

In this paper we have presented the first automated dealiasing algorithm for Doppler velocity fields measured by bistatic receivers. The algorithm is based on the 4DD scheme developed for radial velocity data by James and Houze (2001). Modifications are applied to deal with both the monostatically and the bistatically measured Doppler velocity components as well as irregular data structure - that is, azimuth and elevation angles are recorded for each ray. Three procedures have been added to the 4DD scheme developed by James and Houze (2001). The first interpolates the bistatically measured Doppler velocity onto a spherical coordinate system centered around the monostatic radar and also calculates the apparent velocity component from the threedimensional environmental reference wind field in order to start the initial dealiasing. The second involves irregular data structure. It searches for the nearest data point when comparing the measured and the reference wind field. The third, the internal dealiasing algorithm, 
contributes highly to the success of the 4DD scheme by eliminating many of the difficulties for 4DD when dealiasing small isolated cells. When applying the internal dealiasing algorithm after the dealiasing process itself, isolated cells that initially failed the dealiasing procedure can later be identified and corrected when the area is monitored by at least three receivers. The algorithm requires two dealiased wind components to unfold one Doppler velocity component. The dealiasing status together with the reliability of each Doppler velocity is stored as a quality factor after passing each dealiasing routine of the 4DD. When using internal dealiasing, erroneous data can be detected and expunged at the same time. This case study has shown that the internal dealiasing algorithm is a powerful tool not only for those bistatically measured Doppler velocities limited by a small antenna aperture but also for radial velocity measurements taken at high elevations. Beside dealiasing, the quality of the measurement and the dealiasing process is assessed by calculating the empirical cumulative probability function. The internal dealiasing algorithm can also be applied to monostatic multiple-Doppler radar systems. Again, the area under investigation has to be monitored by at least three monostatic Doppler radars in order to apply the internal dealiasing algorithm.

To observe wind fields over large areas, bistatic receivers are usually set up as a dual-Doppler system and the wind information from several dual-Doppler systems are then combined. With the effective method of using the internal dealiasing algorithm as a means to dealias isolated cells, one should consider it when installing additional receivers, especially when the Nyquist velocity is low and one is investigating rapidly evolving systems. Alternatively, when installing several adjoint dual-Doppler systems, rotating antennas can be used to temporally monitor the target area with three receivers. Those isolated, aliased gates that were dealiased exclusively by the internal dealiasing routine can be flagged and the aliasing interval can then be stored for the next time stage.

The internal dealiasing algorithm can also be extended to the time domain. In this case, when comparing the Doppler velocities of two successive scans, that volume scan having more Doppler velocity information can then be used as reference to dealias the other Doppler velocities.

The examples presented herein attempt to show that by using the modified 4DD scheme, even Doppler velocity measurements with a Nyquist velocity interval of $\pm 8.2 \mathrm{~m} \mathrm{~s}^{-1}$ within downburst-producing thunderstorms can be accomplished. When this occurs, rapid scans have to be performed in order to derive simultaneously microphysical and dynamical parameters in rapidly evolving weather situations. Between a time difference of 5-10 min the dynamical and microphysical structure of a convective cloud can change rapidly, as illustrated in Figs. $7 b$ and $7 d-$ the area south of OP at a range of $50 \mathrm{~km}$. In this case, a convective cell enlarges within 6 min. Furthermore, in the wind field example showing the early stage of developing thunderstorm, we have demonstrated that one can better detect and track the structure, intensity, and development of a downburst measured in real time when using a bistatic Doppler radar network than a monostatic Doppler radar alone.

We also have attempted to prove the comprehensiveness of the modified 4DD scheme when measuring a temporally irregular dataset. If the time difference between two successive volume scans is less than $30 \mathrm{~min}$, it is necessary to derive the three-dimensional environmental wind field for the internal dealiasing either from the previous dealiased velocity field or from a VAD analysis. Then velocity fields sampled with Nyquist intervals of even $\pm 8.2 \mathrm{~m} \mathrm{~s}^{-1}$ can be dealiased by using the modified 4DD. Failure was observed only for single isolated gates, which could later be corrected with the internal dealiasing algorithm.

As a result, monostatically and bistatically measured wind components can be dealiased operationally with the modified 4DD scheme and the horizontal wind vector can be determined. Thus, horizontal wind fields, which are an important factor in meteorological processes, can be used directly for numerous application such as research studies, assimilation into numerical weather prediction models, as well as nowcasting and warning of severe weather at airports and around populated areas.

Acknowledgments. First we would like to thank Curtis James and Robert Houze, at the University of Washington, for providing the 4DD scheme. Thanks are also due to Hans Volkert who arranged the scientific exchange between Météo-France and DLR, and who provided us with help and guidance. Furthermore, we wish to thank Martin Hagen for the fruitful cooperation and enormous support he gave us while operating the bistatic network. We would also like to thank Hermann Scheffold, Hans Krafcyk, and Fred Ritenberg for their technical support at both radar systems. We would also like to express our gratitude to Nikolai Dotzek, Hartmut Höller, and Thorsten Fehr for all their assistance during the VERTIKATOR campaign. Many thanks go to Nerissa Röhrs for help with the English language. We also thank the anonymous reviewers for helpful comments.

\section{REFERENCES}

Bergen, W. R., and S. C. Albers, 1988: Two- and three-dimensional dealiasing of Doppler radar velocities. J. Atmos. Oceanic Technol., 5, 305-319.

Binder, P., and Coauthors, cited 1995: Mesoscale Alpine Programme: Design proposal. MAP Data Centre, Technical Report, ETH Zürich. [Available online at http://www.map.ethz.ch/map-doc/ proposal.htm.]

Bougeault, Ph., and Coauthors, 2001: The MAP special observing period. Bull. Amer. Meteor. Soc., 82, 433-462.

Browning, K. A., and R. Wexler, 1968: The determination of kinematic properties of a wind field using Doppler radar. J. Appl. Meteor., 7, 105-113. 
de Elia, R., 2000: Performance study of a bistatic radar network. Ph.D. thesis, Dept. of Atmospheric and Oceanic Sciences, McGill University, $153 \mathrm{pp}$.

- , and I. Zawadzki, 2000: Sidelobe contamination in bistatic radars. J. Atmos. Oceanic Technol., 17, 1313-1329.

Doviak, J. R., and D. S. Zrnić, 1984: Doppler Radar and Weather Observations. Academic Press, 458 pp.

Friedrich, K., 2002: Determination of three-dimensional wind-vector fields using a bistatic Doppler radar network. Ph.D. thesis, Fakultaet fuer Physik, Ludwig-Maximilians-Universitaet Muenchen, 135 pp. [Available online at http://www.op.dlr.de/ pa4k/.]

— , and M. Hagen, 2004: On the use of advanced Doppler radar techniques to determine horizontal wind-fields for operational weather surveillance. Meteor. Appl., in press.

_ _ - and P. Meischner, 2000: Vector wind field determination by bistatic multiple-Doppler radar. Phys. Chem. Earth, 25B, 1205-1208.

Hoeller, H., V. N. Bringi, J. Hubbert, M. Hagen, and P. F. Meischner, 1994: Life cycle and precipitation formation in a hybrid-type hailstorm revealed by polarimetric and Doppler radar measurements. J. Atmos. Sci., 51, 2500-2522.

James, C. N., and R. A. Houze, 2001: A real-time four-dimensional Doppler dealiasing scheme. J. Atmos. Oceanic Technol., 18, 1674-1683.

Joss, J., and Coauthors, 1998: Final report NFP31: Operational use of radar for precipitation measurements in Switzerland. vdf
Hochschulverlag AG an der ETH Zuerich Technical Report, (ISBN 3728125016 ), 108 pp.

Keeler, R. J., and R. E. Passarelli, 1990: Signal processing for atmospheric radars. Radar in Meteorology, D. Atlas, Ed., Amer. Meteor. Soc., 199-229

Lhermitte, R. M., and D. Atlas, 1961: Precipitation motion by pulse Doppler. Proc. Ninth Weather Radar Conf., Kansas City, MO, Amer. Meteor. Soc., 498-503.

Protat, A., and I. Zawadzki, 1999: A variational method for real-time retrieval of three-dimensional wind field from multiple-Doppler bistatic radar network data. J. Atmos. Oceanic Technol., 16, 432 449

Ray, S. P., K. K. Wagner, K. W. Johnson, J. J. Stephens, W. C. Bumgarner, and E. A. Mueller, 1978: Triple-Doppler observations of a convective storm. J. Appl. Meteor., 17, 1201-1212.

Satoh, S., and J. Wurman, 2003: Accuracy of wind fields observed by a bistatic Doppler radar network. J. Atmos. Oceanic Technol., 20, 1077-1091.

Tabary, P., G. Scialom, and U. Germann, 2001: Real-time retrieval of the wind from aliased velocities measured by Doppler radars. J. Atmos. Oceanic Technol., 18, 875-882.

Takaya, Y., and M. Nakazato, 2002: Error estimation of the synthesized two-dimensional horizontal velocity in a bistatic Doppler radar system. J. Atmos. Oceanic Technol., 19, 74-79.

Wurman, J., S. Heckman, and D. Boccippio, 1993: A bistatic multipleDoppler radar network. J. Appl. Meteor., 32, 1802-1814. 